\title{
Lungenembolie auf der Intensivstation
}

\section{Daniel Dürschmied, Jürgen Heinz, Matthias Siepe, Christoph Bode}

Im Sommer 2014 hat die European Society of Cardiology (ESC) die überarbeitete Leitlinie zur Behandlung der akuten Lungenembolie herausgegeben. Dieser Artikel stellt wichtige Empfehlungen daraus zur Behandlung der häufig vital bedrohten Patienten vor.

\section{Einleitung}

Als Komplikation einer provozierten (z. B. nach orthopädischer Operation) oder unprovozierten (ohne erkennbaren Auslöser) tiefen Venenthrombose droht der Tod durch eine Lungenembolie. Eine Lungenembolie kann einerseits während der Behandlung kritisch kranker Patienten auf der Intensivstation entstehen, andererseits werden akut an einer Lungenembolie erkrankte Patienten häufig primär auf einer Intensivstation behandelt. Daher muss jeder auf einer Intensivstation tätige Arzt mit Pathogenese, Diagnostik und Therapie der Lungenembolie vertraut sein. Im Sommer 2014 hat die European Society of Cardiology (ESC) eine exzellent ausgearbeitete und im Klinikalltag sehr hilfreiche Leitlinie herausgegeben, deren wichtigste Punkte und insbesondere für die Akuttherapie relevante Empfehlungen im Folgenden zusammengefasst werden (Infobox 1) [1].

Nomenklatur. Die semantische Diskussion, ob eine Lungenembolie korrekterweise als „Lungenarterienembolie“ zu bezeichnen wäre, ist eher akademischer Natur und im angelsächsischen Raum weitgehend irrelevant. Der Begriff „Lungenembolie“ ist sowohl international klinisch gebräuchlich als auch korrekt, denn eine Abgrenzung zu einer „Lungenvenenembolie“ die es nicht gibt - oder einer „Bronchialarterienembo-

\section{Infobox 1}

Leitlinienempfehlungen

Die Empfehlungsklassen der Leitlinien der European Society of Cardiology (ESC) und der Deutschen Gesellschaft für Kardiologie (DGK) entsprechen folgender Nomenklatur:

I „soll“ (klare Indikation)

Ila „sollte“ (die Evidenz/Expertenmeinung spricht trotz widersprüchlicher Daten für die Maßnahme)

Ilb „kann“ (Nutzen/Effektivität der Maßnahme ist weniger gut belegt)

III „sollte nicht“ (es wird empfohlen, die Maßnahme zu unterlassen)

Die Evidenzgrade beruhen auf folgenden Grundlagen:

A mehrere randomisierte Studien

$B$ eine randomisierte oder mehrere große, nicht randomisierte Studien

C Konsensusmeinungen von Experten und/oder kleinen Studien, retrospektiven Erhebungen oder Registern

lie“ in den Vasa privata - die spontan nicht klinisch relevant auftritt, sondern lediglich iatrogen bei Lungenblutung als Bronchialarterienembolisation zur Blutstillung angewendet wird - ist nicht nötig. Eine 


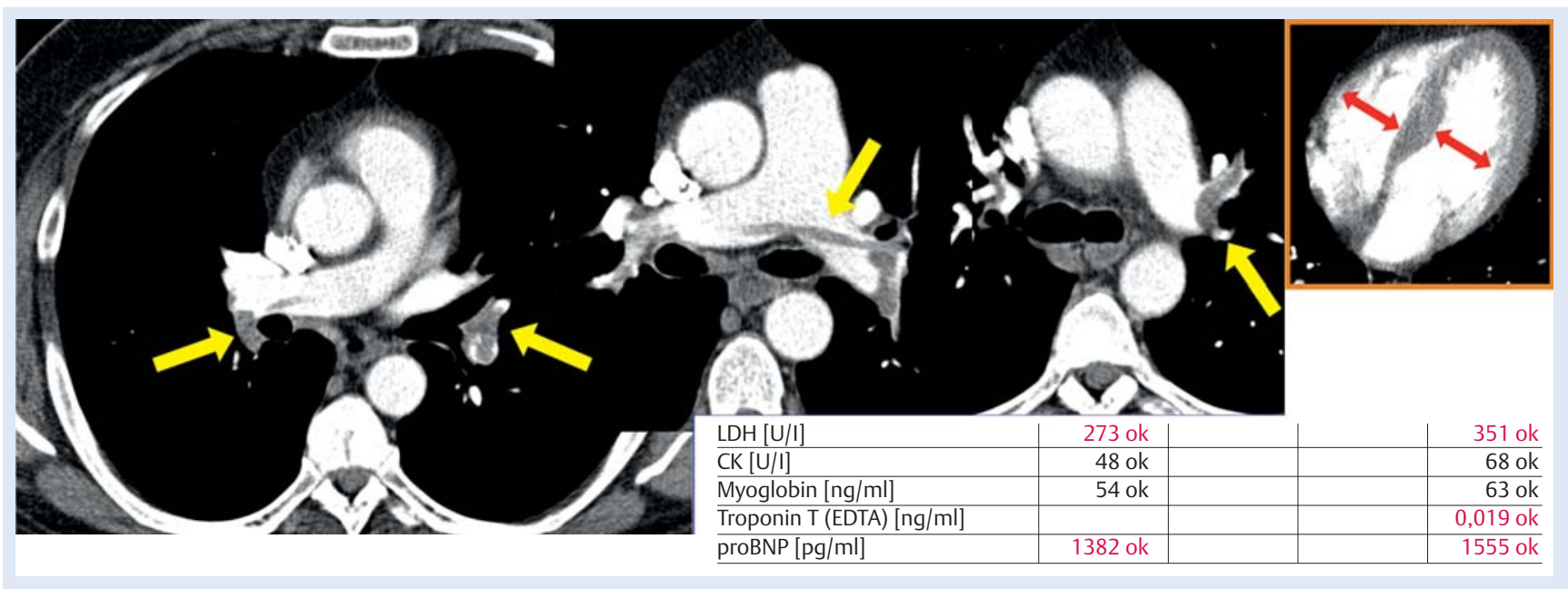

Abb. 1 56-jähriger Patient mit 1 Stunde zuvor aufgetretener Lungenembolie. Beginnende, akute Rechtsherzbelastung im CT und auch laborchemisch fassbar (Troponin T und proBNP leicht erhöht, CK und Myoglobin normwertig). Mehrere Segment-Pulmonalarterien beidseits sind durch einen elongierten Embolus verlegt (gelbe Pfeile), der auf der Pulmonalisbifurkation reitet. Der Durchmesser des rechten Ventrikels übersteigt diskret denjenigen des linken Ventrikels (rote Doppelpfeile). Echokardiografisch liegt noch kein D-Sign vor (Abflachung des Ventrikelseptums - nicht dargestellt). Die Tabelle zeigt die laborchemischen Parameter bei Aufnahme (rechts) und 4 Stunden später (links, rot - außerhalb des Referenzbereichs, ok - validiert).

Lungenembolie betrifft immer die Pulmonalarterien und führt dort zur Verlegung der Strombahn.

Ätiologie. Bei mehr als 20\% der Patienten mit Lungenembolie war die tiefe Venenthrombose klinisch stumm geblieben oder lässt sich keine Emboliequelle mehr nachweisen, weil ein kompletter Venenthrombus embolisierte. In seltenen Fällen embolisiert ein rechtskardialer Thrombus, z. B. bei Vorhofflimmern oder bei rechtskardialen Klappenauflagerungen, der dann aber in der Regel zu kleinen, teils asymptomatischen Lungenembolien führt.

Symptomatik. Für den Patienten kann eine Lungenembolie klinisch stumm verlaufen und erst als Zufallsbefund entdeckt werden (z.B. bei einer Verlaufs-CTUntersuchung bei Tumorpatienten). Das Spektrum der klinischen Erstmanifestationen erstreckt sich über akut bis schleichend einsetzende thorakale Schmerzen, Husten, Stress und Dyspnoe bis hin zum Schock und Herzstillstand.

Therapie. Der wichtigste Baustein der Therapie einer tiefen Venenthrombose ist die plasmatische Antikoagulation in therapeutisch wirksamer Dosierung, um ein fortschreitendes Thrombuswachstum und die Embolisierung zu verhindern.

Hämodynamisch instabile Patienten mit Lungenembolie im Schock weisen mit über 15\% eine enorm hohe 30-Tage-Mortalität auf und bedürfen einer umgehenden Wiedereröffnung der verlegten Lungen- strombahn - in der Regel primär mit einer systemischen Thrombolyse [1]. Besonders schwierig fällt aber oft die Entscheidung, ob bei hämodynamisch stabilen Patienten mit einer Lungenembolie mit mittlerem Risiko die Lungenstrombahn rekanalisiert werden sollte, wenn Zeichen einer akuten Rechtsherzbelastung vorliegen - wie beim in Abb. 1 vorgestellten Patienten. Auch für diese Fälle geben die neuen Leitlinien hilfreiche, evidenzbasierte Entscheidungshilfen.

\section{Risikofaktoren}

Nach wie vor ist die von Rudolf Virchow 1856 postulierte Virchow-Trias die Grundlage für das Verständnis der Pathogenese venöser thromboembolischer Ereignisse. Virchow führte aus, dass eine erhöhte Gerinnbarkeit des Bluts (Hyperkoagulabilität), ein verlangsamter Blutfluss (Stase) und eine Schädigung der Gefäßwand Ursachen für das Entstehen von Thrombosen sind. Die Faktoren, die zu den genannten Veränderungen führen, sind mannigfaltig und viele dieser Faktoren sind bei Intensivpatienten vorhanden. Da eine Lungenembolie in bis zu 90\% der Fälle ihren Ursprung in einer tiefen Beinvenenthrombose hat, geht man von gleichartigen Risikofaktoren für eine Lungenembolie wie für eine tiefe Beinvenenthrombose aus. Im Folgenden wird daher nicht zwischen Risikofaktoren für eine Lungenembolie oder eine tiefe Beinvenenthrombose unterschieden, sondern es werden einheitlich Risikofaktoren für thromboembolische Ereignisse genannt. 


\section{Tabelle 1}

Hereditäre Thrombophilien. VTE: venöse Thromboembolie.

\begin{tabular}{|c|c|c|c|}
\hline Gerinnungsstörung & Prävalenz & Relatives Risiko & Anteil bei Patienten mit VTE \\
\hline Faktor-V-Leiden-Mutation (heterozygot) & $3-7 \%$ & $5-8 \%$ & $20-40 \%$ \\
\hline Faktor-V-Leiden-Mutation (homozygot) & $0,02 \%$ & $40-50 \%$ & $3 \%$ \\
\hline Faktor-II-Mutation (heterozygot) & $1-3 \%$ & $3 \%$ & $7-16 \%$ \\
\hline Faktor-V-Leiden- u. Faktor-II-Mutation (heterozygot) & $<0,05 \%$ & $20 \%$ & $2,3 \%$ \\
\hline Protein-S-Mangel (heterozygot) & $0,7-2,3 \%$ & $5-11 \%$ & $2-4 \%$ \\
\hline Protein-C-Mangel (heterozygot) & $0,2-0,5 \%$ & $7-10 \%$ & $4-5 \%$ \\
\hline Antithrombin-Mangel (heterozygot) & $0,1-0,3 \%$ & $4-50 \%$ & $1,5-3 \%$ \\
\hline
\end{tabular}

Bei den Risikofaktoren unterscheidet man angeborene (hereditäre Thrombophilie) von erworbenen Risikofaktoren, die erst im Laufe des Lebens erworben werden. Diese können zeitlich begrenzt oder permanent vorhanden sein. In den meisten Fällen müssen allerdings mehrere Faktoren zusammenkommen, damit ein thromboembolisches Ereignis auftritt. Die venöse Thromboembolie ist somit ein typisches Beispiel für eine multifaktorielle Erkrankung.

Angeborene Risikofaktoren. Die hereditäre Thrombophilie ist definiert als genetisch determinierte Neigung zu Thromboembolien. Zu den angeborenen Risikofaktoren zählen die in Tab. 1 aufgeführten Veränderungen, welche mit einem unterschiedlich hohen Risiko für thromboembolische Ereignisse einhergehen und in unterschiedlicher Prävalenz in der Bevölkerung auftreten, wobei die Angaben in der Literatur teils erheblich variieren.

Von der häufigsten hereditären Thrombophilie der heterozygoten Faktor-V-Leiden-Mutation - sind etwa $5 \%$ der Bevölkerung betroffen. Das relative Risiko dieser Personen für eine venöse Thromboembolie liegt ebenfalls bei etwa $5 \%$.

Die häufigsten beiden Veränderungen, die bei Patienten mit venöser Thromboembolie nachweisbar sind, sind die APC-Resistenz, deren häufigste Ursache die Faktor-V-Leiden-Mutation ist, und die ProthrombinMutation (Faktor-II-Mutation). Beide Veränderungen sind bei 50-60\% der Patienten mit thromboembolischen Ereignissen nachzuweisen. Unter bestimmten Bedingungen kann eine APC-Resistenz auch erworben sein (z. B. in der Schwangerschaft oder unter Einnahme von Ovulationshemmern), wobei die klinische Bedeutung dieser Veränderung nicht klar ist.

Seltenere angeborene Veränderungen sind der Mangel an Protein S, Protein C oder Antithrombin sowie die sehr seltenen Fälle eine Dysfibrinogenämie. Der hereditäre Antithrombinmangel weist dabei das höchste Risiko für eine venöse Thromboembolie auf, während die Faktor-II- und -V-Mutationen mit einem geringeren Risiko für Thromboembolien einhergehen, ähnlich dem Risiko für Thromboembolien während einer Schwangerschaft.

Zusätzlich zu den genannten Ursachen hat sich in den letzten Jahren gezeigt, dass ein permanent erhöhter Faktor VIII mit einem ca. 5-fach erhöhten Thromboserisiko assoziiert ist, wobei transitorische Erhöhungen (z.B. im Rahmen einer Akut-Phase-Reaktion) ausgeschlossen werden müssen. Insbesondere das Rezidivrisiko für eine venöse Thromboembolie scheint bei Patienten mit einem persistierend erhöhtem Faktor VIII deutlich erhöht zu sein [2].

Auf mögliche weitere hereditäre Ursachen, die mit einem erhöhten Risiko für thromboembolische Ereignisse in Verbindung gebracht werden, wird aufgrund der Seltenheit der Veränderungen und des fraglichen Zusammenhangs nicht näher eingegangen.

Ein Screening auf eine hereditäre Thrombophilie ist bei einem thromboembolischen Ereignis bei Patienten auf der Intensivstation nicht sinnvoll, da im Rahmen des akuten Ereignisses verschiedene Marker verändert sein 


\section{Infobox 2}

Erworbene Risikofaktoren für Thromboembolien

- Alter

- frühere thromboembolische Ereignisse

- Immobilisierung, Bettlägrigkeit, Lähmung

- Trauma

- Operation

- Malignom

- hämatologische Erkrankungen

- Polyzythaemia vera

- essenzielle Thrombozythämie

- paroxysmale nächtliche Hämoglobinurie

- Schwangerschaft und Wochenbett

- zentralvenöser Katheter

- internistische Erkrankungen

- akuter Herzinfarkt

- Herzinsuffizienz

- respiratorische Insuffizienz

- Infektionen

- chronische entzündliche Darmerkrankungen
- nephrotisches Syndrom

- chronische Lebererkrankungen

- Morbus Behçet

- Medikamente

- orale östrogenhaltige Kontrazeptiva

- Hormonersatztherapie

- Tamoxifen

- Thalidomid, Lenalidomid

- Glukokortikoide

- Bevacizumab

- intravenöser Drogenabusus

- Heparin (heparininduzierte Thrombozytopenie)

- Chemotherapie

- Antiphospholipid-Syndrom (APS)

- anatomische Anomalien

- Paget-Schroetter-Syndrom

- May-Thurner-Syndrom

- Abnormitäten der V. cava inferior (Agenesie, Hypoplasie, Malformation)

- Übergewicht

können und somit nicht verwertbar sind (z. B. Protein S, Protein C, Antithrombin) und andererseits der Nachweis einer Thrombophilie keine therapeutischen Konsequenzen zur Folge hat.

Erworbene Risikofaktoren. Erworbene Risikofaktoren oder prädisponierende Faktoren für Thromboembolien sind mannigfaltig, wobei den bedeutendsten Risikofaktor das höhere Lebensalter darstellt. Durch ein höheres Lebensalter wird das allgemeine Thromboserisiko, das durch hereditäre oder erworbene Risikofaktoren bereits vorhanden ist, durch Interaktion der verschiedenen Risikofaktoren im Sinne der Multikausalität erhöht. Eine Übersicht über weitere erworbene Risikofaktoren gibt Infobox $\mathbf{2}$ ohne Anspruch auf Vollständigkeit.

In 2 unabhängigen Studien war bei Intensivpatienten das Risiko für eine Lungenembolie durch folgende Faktoren erhöht $[3,4]$ :

- schwere exazerbierte COPD

- akute internistische Erkrankung

- meningeale Blutung

- Fraktur der Wirbelsäule

- schwere Hypoxämie

- Fehlen einer medikamentösen Thromboseprophylaxe

Ein Screening auf eine hereditäre Thrombophilie ist bei einem thromboembolischen Ereignis bei Intensivpatienten nicht sinnvoll. Eine Vielzahl der typischen Charakteristika von Intensivpatienten erhöht ihr Risiko für eine venöse Thromboembolie.

\section{Heparininduzierte Thrombozytopenie}

Während das Screening auf eine hereditäre Thrombophilie beim Akutereignis nicht sinnvoll ist, muss man beim Verdacht auf eine heparininduzierte Thrombozytopenie (HIT) eine rasche Abklärung veranlassen, da die sofortige Beendigung der Heparingabe und der Umstieg auf ein alternatives Antikoagulans lebensrettend für den Patienten sein kann. Auch wenn die Inzidenz der HIT mit 0,3-0,5\% bei Intensivpatienten niedrig ist, kann das Übersehen einer HIT fatale Folgen haben [5]. In der Literatur wird eine Amputationsrate von bis zu 6\%, eine Mortalität von bis zu 7\% und eine Lungenembolie in bis zu 25\% der Fälle einer HIT berichtet.

Symptomatik. Der Verdacht auf eine HIT ergibt sich u.a. bei folgenden klinischen Auffälligkeiten:

- Thrombozytopenie oder Thrombozytenabfall um 50\% vom Ausgangswert ab dem vierten Tag nach Beginn der Heparintherapie (cave: auch bei Reexposition innerhalb von 30 Tagen durch noch vorhandene Antikörper auch innerhalb von 24 Stunden möglich)

- Progression eines thromboembolischen Ereignisses trotz Heparingabe

- arterielle Gefäßverschlüsse

- Hautveränderungen an Einstichstellen der Heparininjektionen (z. B. Hautnekrosen, Quaddeln).

Diagnostik. Da eine Thrombozytopenie gerade bei Intensivpatienten nicht selten ist, sollte man die klinische Wahrscheinlichkeit mit verschiedenen ScoreSystemen ermitteln (z.B. 4T-Score, HEP-Score) sowie die Diagnose rasch bestätigen oder ausschließen (Antigen-Test und ggf. Bestätigung durch einen funktionellen Test). Erwähnt werden muss allerdings, dass z.B. bei bis zu $50 \%$ der kardiochirurgischen Patienten HIT-Antikörper nachgewiesen werden, aber nur 1-2\% dieser Patienten thrombotische Gefäßverschlüsse entwickeln [6]. 


\section{Primärprophylaxe}

Aufgrund des hohen Risikos für thromboembolische Ereignisse bei Intensivpatienten mit einer nicht unerheblichen Letalität bei einer Lungenembolie ist eine entsprechende Thromboseprophylaxe von enormer Bedeutung. Wann immer möglich sollte man unter Abwägung des Nutzens und des Risikos (Prophylaxe vs. Blutungsrisiko) eine medikamentöse Thromboseprophylaxe durchführen. Aufgrund der Besonderheiten bei Intensivpatienten erhalten diese häufig weiterhin unfraktioniertes Heparin als kontinuierliche intravenöse Gabe. Gründe hierfür sind:

- kurze Halbwertszeit bei i.v. Gabe, sodass Interventionen nach Unterbrechung der i.v. Gabe rasch durchgeführt werden können

- einfachere Antagonisierung bei Blutungen

- nicht so ausgeprägte Akkumulation bei Niereninsuffizienz

Grundsätzlich kann man aber auch bei Intensivpatienten niedermolekulare Heparine und Fondaparinux einsetzen, wobei bei Niereninsuffizienz eine entsprechende Dosisanpassung erforderlich ist. Bei Patienten mit einem hohem Blutungsrisiko oder schwerer Thrombozytopenie $(<20000 / \mu \mathrm{l})$ sind physikalische Maßnahmen als Alternative zur medikamentösen Antikoagulation zu erwägen, auch wenn ihr Nutzen zum Teil kritisch diskutiert wird.

\section{Risikobeurteilung}

Unabhängig von Größe und Lokalisation des Embolus in der Bildgebung (meist CT) muss man sofort eine Einschätzung des frühen Mortalitätsrisikos vornehmen, denn hiervon hängt das weitere Vorgehen ab [1]. Mit sehr einfachen und schnell verfügbaren Mitteln kann der behandelnde Arzt innerhalb weniger Minuten einschätzen, ob eine Hoch-, Intermediär- oder Niedrigrisiko-Lungenembolie vorliegt (Tab.2):

- Bei Hochrisiko-Lungenembolie dominiert klinisch die hämodynamische Instabilität. Wenn die Lungenstrombahn nicht umgehend rekanalisiert wird, liegt die 30-Tage-Letalität bei über 15\% (in einigen Studien sogar bis zu 65\%).

- Bei Intermediärrisiko-Lungenembolie liegt die frühe Sterblichkeit zwischen 3 und 15\%. Die aktuellen Leitlinien können erstmals - gestützt auf aktuelle Studien - Intermediärrisiko-Patienten definieren, die von einer Rekanalisierung profitieren (bei akuter Rechtsherzbelastung, myokardialer Ischämie und niedrigem Blutungsrisiko; s. u.).

- Niedrigrisiko-Patienten sollten nicht rekanalisiert werden und können in vielen Fällen früh ambulant behandelt werden.

Pulmonary Embolism Severity Index. Eine feinere Risikobeurteilung erlaubt der Pulmonary Embolism Severity Index (PESI), der anhand 11 klinischer Charakteristika 5 Risikoklassen unterscheidet [1,7]. Zu

\section{Tabelle 2}

Risikobeurteilung und risikoadaptierte Therapie der Lungenembolie.

\begin{tabular}{|c|c|c|c|c|}
\hline \multirow{2}{*}{$\begin{array}{l}\text { 30-Tage- } \\
\text { Mortalitätsrisiko }\end{array}$} & \multicolumn{3}{|c|}{ Risikomarker } & \multirow[t]{2}{*}{ Therapie } \\
\hline & Schock & $\begin{array}{l}\text { akute Rechts- } \\
\text { herzbelastung }\end{array}$ & $\begin{array}{l}\text { myokardiale Nekrose } \\
\text { (Troponin) }\end{array}$ & \\
\hline hoch (>15\%) & + & + & irrelevant & $\begin{array}{l}\text { - UFH } \\
\text { - Thrombolyse } \\
\text { - ggf. Embolektomie } \\
\text { - nach Stabilisierung NMH/Fondaparinux + VKA oder DOAK }\end{array}$ \\
\hline \multirow[t]{3}{*}{ intermediär (3-15\%) } & - & + & + & $\begin{array}{l}\text { - UFH + Thrombolyse (ggf. Embolektomie) erwägen } \\
\text { - NMH/Fondaparinux+VKA } \\
\text { - DOAK }\end{array}$ \\
\hline & - & + & - & $\begin{array}{l}\text { - NMH/Fondaparinux + VKA } \\
\text { - DOAK }\end{array}$ \\
\hline & - & - & + & \\
\hline niedrig $(<1 \%)$ & - & - & - & $\begin{array}{l}\text { - NMH/Fondaparinux + VKA } \\
\text { - DOAK }\end{array}$ \\
\hline
\end{tabular}

DOAK: direkte orale Antikoagulanzien; NMH: niedermolekulares Heparin; UFH: unfraktioniertes Heparin; VKA: Vitamin-K-Antagonisten 
den Charakteristika zählen u.a. Alter, Herz- und Atemfrequenz, malignes oder kardiopulmonales Grundleiden, Vigilanz- oder Oxygenierungsstörung. Für die beiden niedrigsten PESI-Klassen (Frühletalität 0 - 1,6\% und 1,7-3,5\%) erlauben die Leitlinien eine ambulante Behandlung mit einer Klasse-IlaB-Empfehlung.

\section{Diagnostik}

Symptomatik und Labor. Typische Symptome sind Dyspnoe, thorakale Schmerzen, Husten, Fieber, Hämoptysen, Synkope und Symptome einer tiefen Venenthrombose. Die klinische Vortestwahrscheinlichkeit für eine venöse Thromboembolie kann mit dem Wells- oder dem Genfer Score ermittelt werden. Laborchemisch kann die Bestimmung der D-Dimere sehr hilfreich sein, weil mit einem assayabhängigen, negativ prädiktiven Wert von über 95\% eine venöse Thromboembolie recht sicher ausgeschlossen werden kann (3-Monats-Risiko für eine venöse Thromboembolie zwischen 0 und 0,4\%).

Bei hoher Vortestwahrscheinlichkeit und typischen Symptomen sollte man eine weiterführende Diagnostik durchführen, weil auch negative D-Dimere keine absolute Sicherheit bieten können.

Als Marker der myokardialen Schädigung bei akuter Rechtsherzbelastung dienen im Wesentlichen Troponine und BNP oder NT-proBNP. Der frühe Marker „hearttype fatty acid-binding protein“ (H-FABP) hat wie das NT-proBNP einen hohen negativ prädiktiven Wert von 99\% für die 30-Tage-Mortalität.

CT-Angiografie. Goldstandard für die Darstellung einer Lungenembolie ist die Multidetektor-CT-Angiografie mit Kontrastmittel.

Ventilations-Perfusions-Szintigrafie. Die Stärken der alternativ anwendbaren Ventilations-PerfusionsSzintigrafie sind die geringere Strahlenbelastung (ca. $1,1 \mathrm{mSv}$ vs. $2-6 \mathrm{mSv}$ bei der CT), die hohe Sensitivität (ein normales Szintigramm schließt eine Lungenembolie mit hinreichender Sicherheit aus) und die fast fehlende Nephrotoxizität und Allergenität.

Pulmonalisangiografie. Die invasive Pulmonalisangiografie bleibt wegen des hohen Komplikationsrisikos Notfällen vorbehalten - wenn etwa im Herzkatheterlabor reanimiert wird (IIbC-Empfehlung). Immerhin kann man damit innerhalb von Minuten transarteriell eine KHK und Aortendissektion sowie transvenös eine
Lungenembolie als Ursache eines Herzstillstands ausschließen bzw. diagnostizieren.

MRT. Die Magnetresonanztomografie hat in der Lungenemboliediagnostik noch nicht Einzug in die klinische Anwendung gefunden.

Echokardiografie. Bei allen Patienten mit vermuteter oder nachgewiesener Lungenembolie und hohem oder intermediärem Risiko sollte man unbedingt eine Echokardiografie durchführen. Mit ihr kann man eine rechtsventrikuläre Dysfunktion als Zeichen einer akuten Rechtsherzbelastung nachweisen (u. a. Septumabflachung als D-Sign, rechtsventrikuläre Erweiterung). Nicht zweifelsfrei ausschließen lassen sich akute Anteile bei chronischer Rechtsherzbelastung mit einer globalen Reduktion der systolischen rechtsventrikulären Pumpfunktion (bei akuter Rechtsherzbelastung zumindest noch erhaltene apikale Kontraktilität) und ggf. einer Abnahme der rechtsventrikulären Wanddurchmesser. Der direkte Nachweis von echogenen, teilmobilen Strukturen im rechten Herzen oder den Pulmonalarterien erleichtert die Diagnose (auch mit transösophagealer Echokardiografie möglich).

Venöse Kompressionssonografie. Die venöse Kompressionssonografie kann eine tiefe Venenthrombose als Ursprung der Lungenembolie sicher diagnostizieren.

\section{Therapie}

Patienten mit Lungenembolie, die auf einer Intensivstation behandelt werden, haben meist ein hohes oder intermediäres frühes Todesrisiko aufgrund der Lungenembolie (Tab. 2). Ist bei hämodynamischer Instabilität eine CT nicht unmittelbar möglich, kann auch der echokardiografische Nachweis einer akuten Rechtsherzbelastung eine Thrombolyse rechtfertigen. Andererseits erlaubt das Fehlen dieser echokardiografischen Zeichen den Ausschluss einer Lungenembolie als Schockursache. Sind bei Instabilität weder Echokardiografie noch CT verfügbar oder bei präklinischer Reanimation nicht möglich, kann auch die Verdachtsdiagnose „Lungenembolie“ eine Thrombolyse rechtfertigen.

\section{Hochrisikopatienten}

Volumengabe. Zur hämodynamischen Stabilisierung ist eine milde Volumengabe (500 ml Kristalloidlösung) möglich. 
Eine massive Volumentherapie kann die rechtsventrikuläre Funktion durch Overstretching weiter verschlechtern.

Katecholamine. Katecholamine der Wahl im refraktären Schock sind Adrenalin und Noradrenalin. Bei reduziertem Herzindex kann man unter Beachtung der vasodilatierenden Wirkung bei einigen Patienten Dobutamin geben. Pathophysiologisch sinnvoll erscheint auch Levosimendan, für das es bei Lungenembolie jedoch bislang nur eine unzureichende Evidenz gibt (nur Fallserien) [1]. Primär sollte man das hypoxische respiratorische Versagen mit einer Sauerstoffinsufflation behandeln. Ein persistierendes Foramen ovale oder ein atrialer Septumdefekt können bei Rechts-links-Shunt die Oxygenierungsstörung verschlechtern. Ist eine nicht invasive oder invasive Beatmung erforderlich, muss man darauf achten, den positiven endexspiratorischen (PEEP) und endinspiratorischen Druck möglichst gering zu halten, um das Preload möglichst wenig zu reduzieren. Eine extrakorporale Herz- oder Lungenunterstützung mit ECMO oder ECLS kann in refraktären Fällen lebensrettend sein.

Unfraktioniertes Heparin. Alle Hochrisikopatienten sollte man mit unfraktioniertem Heparin in therapeutischer Dosierung (Ziel: 1,5- bis 2,5-fache aPTT-Verlängerung) antikoagulieren (Klasse-IC-Empfehlung).

Thrombolyse/Embolektomie. Daneben ist unverzüglich eine systemische Thrombolyse (IB-Empfehlung) oder wenn die Lyse absolut kontraindiziert oder frustran verlaufen ist - eine chirurgische Embolektomie erforderlich (IC-Empfehlung). Nur wenn auch die chirurgische Embolektomie nicht möglich ist, sollte man kathetergestützte Verfahren einsetzen (IlaC-Empfehlung).

In über 90\% der Fälle führt eine systemische Thrombolyse zur raschen hämodynamischen Stabilisierung. Wird die Initialphase überlebt, bildet sich die akute Rechtsherzbelastung sowohl unter unfraktioniertem Heparin als auch nach zusätzlicher Lyse meist innerhalb von 7 Tagen zurück.

In Deutschland setzt man zur Lyse meist Alteplase ein (rt-PA, Actilyse): Zunächst wird ein Bolus von $10 \mathrm{mg}$ injiziert, gefolgt von einer Perfusorinfusion von $90 \mathrm{mg}$ über 2 Stunden (Tab. 3). Eine Halbierung der Alteplasedosis auf $50 \mathrm{mg}$ war in 2 kleineren Studien ähnlich effektiv, aber sicherer als die Standarddosierung von $100 \mathrm{mg}$ [1]. Bislang geben die Leitlinien jedoch keine

\section{Tabelle 3}

Systemische (intravenöse) Thrombolyse.

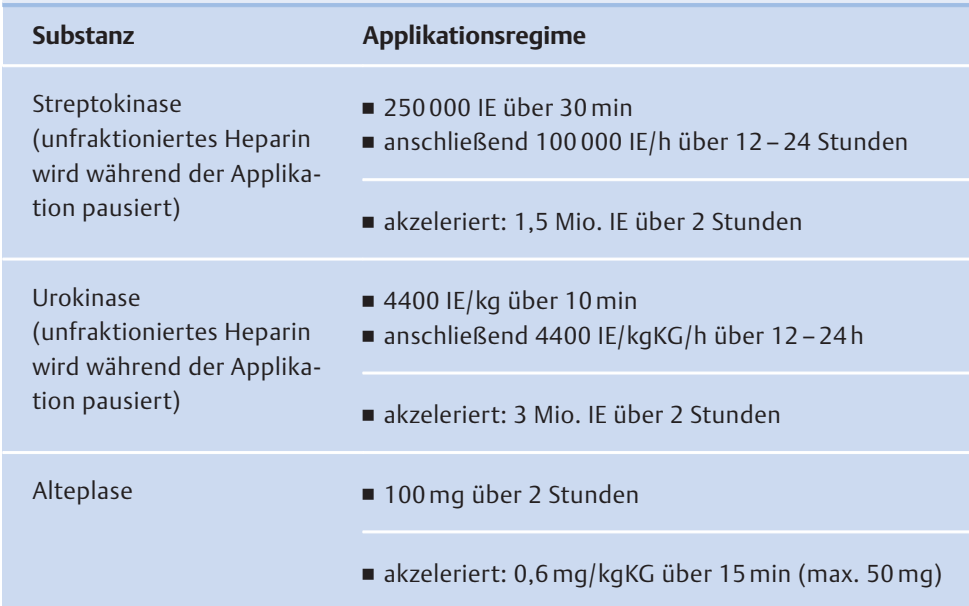

Präferenz für das eine oder andere Regime. Die Niedrigdosis-Alteplasegabe erfolgt akzeleriert über $15 \mathrm{~min}$ $(0,6 \mathrm{mg} / \mathrm{kgKG}$, Maximaldosis $50 \mathrm{mg})$. Insbesondere für blutungsgefährdete Patienten erscheint dieses Regime praktikabel.

Tenecteplase (TNK-tPA, 30-50 mg Bolus über 5-10s gewichtsadaptiert) ist wie auch Reteplase und Desmetoplase zur Therapie der Lungenembolie in Deutschland bislang nicht zugelassen.

Infobox 3 listet absolute und relative Kontraindikationen für eine systemische Lyse auf. Wird eine Thrombolyse jedoch als ultima ratio bei erfolgloser Reanimation bei vermuteter Lungenembolie durchgeführt oder lässt sich keine hämodynamische Stabilisierung bis zur Durchführung einer chirurgischen oder interventionellen Embolektomie erreichen (bzw. ist eine ECLS nicht verfügbar), gelten auch absolute Kontraindikationen nur noch als relativ.

\section{Intermediärrisiko-Patienten}

Bei Intermediärrisiko-Patienten mit akuter Rechtsherzbelastung und einer Erhöhung der myokardialen Nekroseparameter sollte man die Möglichkeit einer systemischen Lyse prüfen, sofern das Blutungsrisiko gering erscheint (IlaB-Empfehlung). Ist die Lyse nicht möglich oder frustran verlaufen, kann man eine chirurgische (IIbC-) oder interventionelle Embolektomie (IIbB-Empfehlung) erwägen. Rationale der blutungsaffinen Lyse ist die mit bis zu 15\% hohe Frühletalität der Intermediärrisiko-Patienten. 


\section{Infobox 3}

Kontraindikationen für eine systemische Thrombolyse

absolute Kontraindikationen:

- Hirnblutung

- ischämischer Insult vor weniger als 6 Monaten

- ZNS-Malignom

- schweres Trauma, Operation oder Schädel-Hirn-Trauma vor weniger als 3 Wochen

- gastrointestinale Blutung vor weniger als 1 Monat

- bekannte Blutungsneigung relative Kontraindikationen:

- TIA vor weniger als 6 Monaten

- orale Antikoagulation

- Schwangerschaft oder erste postpartale Woche

- nicht komprimierbare Punktionsstelle

- traumatische Reanimation

- refraktäre Hypertonie (systolischer Blutdruck über $180 \mathrm{mmHg}$ )

- fortgeschrittene Lebererkrankung

- infektiöse Endokarditis

- aktives Magenulkus
Zusammenfassend lässt sich mit einer systemischen Thrombolyse das Mortalitätsrisiko deutlich senken und ist für jüngere Patienten mit relativ geringem Blutungsrisiko sinnvoll, weil der klinische Nettonutzen überwiegt. Das akzelerierte, dosishalbierte Alteplaseregime wurde zwar in PEITHO nicht untersucht, erscheint aber pathophysiologisch sinnvoll und ist durch die Leitlinien gedeckt. Alternativ kann auch die volle Alteplasedosierung nach Standardregime nach Einzelfallabwägung gegeben werden. Auch bei Intermediärrisiko-Patienten sollte man eine begleitende Antikoagulation mit unfraktioniertem Heparin durchführen.

Die Indikation zur Thrombolyse bei Intermediärrisiko-Patienten sollte man sorgfältig abwägen. Sie kann aber bei jüngeren Patienten mit niedrigem Blutungsrisiko das Überleben deutlich verbessern.
Die im Frühjahr 2014 publizierte PEITHO-Studie zeigte bei 1006 Intermediärrisiko-Patienten eine signifikante Halbierung des primären Endpunkts „Tod oder hämodynamische Dekompensation“ (Abb.2). Signifikant vervielfacht wurden jedoch Blutungskomplikationen. Intrakranielle Blutungen wurden durch die Verabreichung von Tenecteplase von $0,2 \%$ auf 2,0\% erhöht. Diese deutliche Risikoerhöhung im Gesamtkollektiv erscheint inakzeptabel hoch. Die Subgruppenanalyse zeigte jedoch, dass jüngere Patienten (unter 75 Jahre) mit einer relativen Risikoreduktion für den primären Endpunkt um 67\% besonders deutlich profitierten und ihr Blutungsrisiko nicht so stark erhöht war wie das der älteren Patienten.

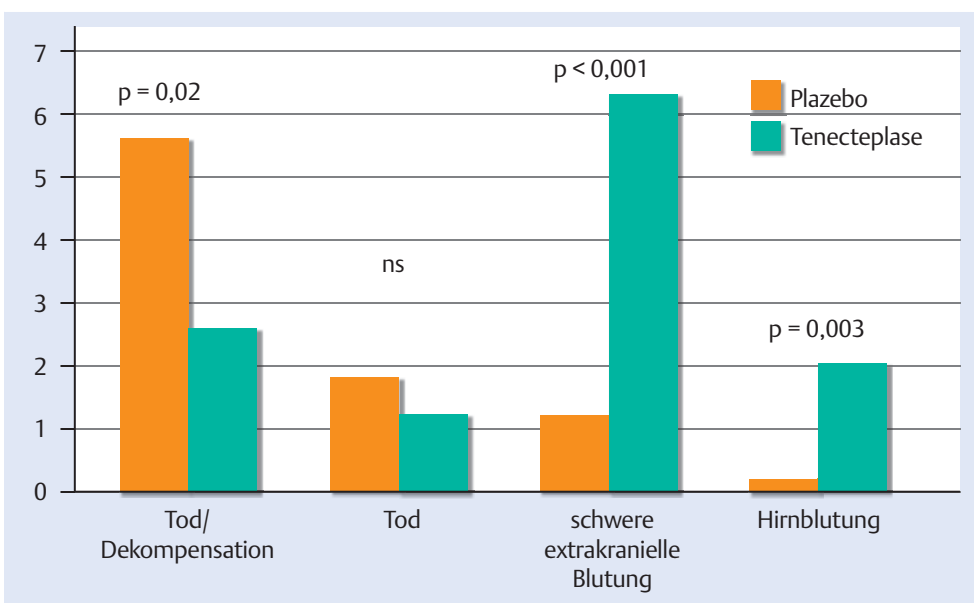

Abb. 2 Relevante Endpunkte im Gesamtkollektiv der PEITHO-Studie, die Effekte der systemischen Thrombolyse bei Patienten mit Intermediärrisiko-Lungenembolie mit akuter Rechtsherzbelastung untersuchte (nach [31]).

\section{Chirurgische und inter- ventionelle Behandlung}

Bei Hochrisiko- oder Intermediärrisiko-Lungenembolie mit rechtsventrikulärer Dysfunktion ist eine chirurgische oder interventionelle Therapie insbesondere dann indiziert, wenn eine Lyse oder Antikoagulation nicht möglich ist oder nicht erfolgreich war. Für die Hochrisiko-Lungenembolie liegt in diesen Fällen eine Klasse-IC-Empfehlung vor [1]. Der Zustand des Patienten diktiert hierbei die Notwendigkeit und Geschwindigkeit des Vorgehens.

Zunächst sollte man alle Maßnahmen unternehmen, um den Patienten hämodynamisch und respiratorisch zu stabilisieren. Kommt es trotz medikamentöser Therapie nicht zu einer Stabilisierung der Hämodynamik, sollte man eine Hypoperfusion des Patienten nicht tolerieren, sondern ein extrakorporales Unterstützungssystem (ECLS) einsetzen. Dies ist in der Notfallsituation über den Zugang der Leistengefäße per Punktion oder mit chirurgischer Freilegung der Gefäße möglich. Ist der Patient hingegen hämodynamisch stabil genug, ist eine operative oder interventionelle Embolektomie möglich. Ob eine chirurgische oder katheterbasierte Embolektomie in Erwägung gezogen wird, hängt insbesondere von der Verfügbarkeit und Expertise ab. Ein direkter Vergleich der beiden Methoden wurde bislang nicht durchgeführt, Daten zur Effektivität beider Therapieformen sind nur begrenzt verfügbar. 


\section{Chirurgische Embolektomie}

Die chirurgische Embolektomie kann lediglich in einem großen Zentrum mit Herz-Lungen-Maschine und herzchirurgischem Personal durchgeführt werden. Aufgrund der Schwere der Erkrankung ist dieser Eingriff insbesondere bei älteren Patienten mit einer hohen Sterblichkeit verbunden (bis zu $46 \%$ bei den über 80 Jährigen) [8]. Neben der Klasse-IC-Empfehlung für die chirurgische Embolektomie bei kontraindizierter oder fehlgeschlagener Thrombolyse kann in Einzelfällen außerhalb der Leitlinienempfehlung auch dann eine chirurgische Therapie erwogen werden, wenn ein großer Thrombus im Foramen ovale, im rechten Herzen oder dem Pulmonalarterienhauptstamm durch Echokardiografie oder CT gesichert wurde [9].

Einschränkend liegt zur chirurgischen Embolektomie allerdings im Vergleich zur thrombolytischen Therapie nur eine ungenügende Evidenz vor. In einer Kohortenstudie mit 40 Höchstrisiko-Patienten konnte die chirurgische Embolektomie erneute Lungenembolien und schwere Komplikationen wie Massenblutung und Todesfälle im Vergleich zur wiederholten Lyse numerisch reduzieren, nachdem eine initiale Lyse erfolglos geblieben war [9]. Während der chirurgischen pulmonalen Embolektomie leitet eine intraoperative transösophageale Echokardiografie den Operateur. Diese beeinflusste in einer systematischen Untersuchung das chirurgische Vorgehen bei 10\% der Patienten [10].

Das chirurgische Vorgehen sieht in den meisten Fällen den Anschluss an die Herz-Lungen-Maschine in bikavaler Technik vor, denn die Thrombektomie kann dann am schlagenden Herzen durchgeführt werden. Hierzu wird die Pulmonalarterie meist im Truncus pulmonalis eröffnet, anschließend werden mit chirurgischem Instrument, großen Embolektomiekathetern oder mit manueller Kompression der Lungenflügel die Thromben geborgen. Ziel der Therapie ist eine möglichst vollständige Entfernung der Thromben (Abb.3).

\section{Das Outcome nach chirurgischer Embolektomie} bestimmt hauptsächlich der präoperative Zustand des Patienten. Ist er vor der chirurgischen Therapie zwar hämodynamisch instabil, aber es lag kein Herzstillstand vor, so zeigen ältere Untersuchungen eine Überlebenswahrscheinlichkeit von über 95\% [11]. Die Sterblichkeit bei Patienten, die zuvor einen Herzstillstand erlitten hatten, ist hingegen deutlich höher $[11-14]$.

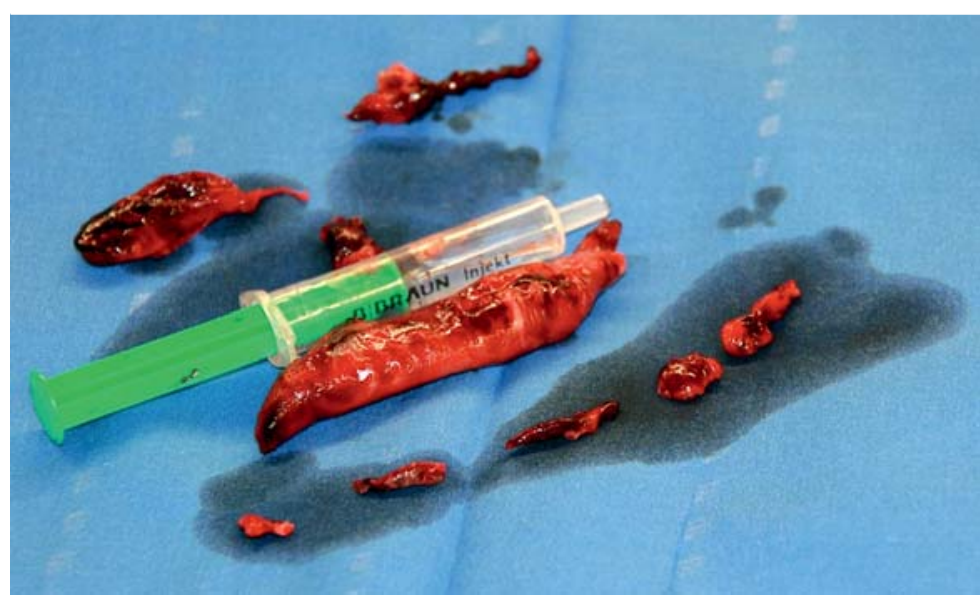

Abb. 3 Frische, mit chirurgischer Embolektomie geborgene Emboli im Größenvergleich mit einer 5-ml-Spritze.

Zusammengefasst ist die chirurgische Embolektomie die Therapie der Wahl für Patienten mit HochrisikoLungenembolie, die nicht lysiert werden können (bei Vorliegen absoluter Kontraindikationen) oder wenn eine Lyse fehlgeschlagen ist. Der Präsident des North Atlantic Thrombosis Forum (NATF) Samuel Goldhaber vom Brigham and Women's Hospital in Boston, USA, rät dringend zur Etablierung multidisziplinärer Teams an den großen Zentren unter Einbeziehung von in der Embolektomie erfahrenen Herzchirurgen. Ähnlich der Heart Teams können auf diese Weise schnell fundierte Entscheidungen getroffen und umgesetzt werden, die dem individuellen Risiko dieser vital bedrohten Patienten gerecht werden.

Die chirurgische Embolektomie muss als Alternative bei Hochrisikopatienten sehr früh in Betracht gezogen werden, wenn eine Thrombolyse nicht möglich oder nicht erfolgreich ist (Klasse-IC-Empfehlung). Sie kann das Leben der akut bedrohten Patienten retten und rechtfertigt auch die notfallmäßige Verlegung in ein Zentrum mit erfahrenen Herzchirurgen.

\section{Interventionelle Embolektomie und lokale Thrombolyse}

Zur interventionellen Embolektomie sind verschiedene Methoden etabliert und bereits klinisch im Einsatz, obwohl sie bislang nur in kleinen Serien untersucht wurden und bislang noch nicht mit anderen Techniken in randomisierter Form verglichen wurden. Wegen der bislang noch hohen Komplikationsraten betonen die Leitlinien den höheren Stellenwert der chirurgischen Embolektomie [1]. 
Rheolytische Embolektomie. Über eine großlumige, venös gelegte Schleuse wird mit einem speziellen Katheter unter Druck Kochsalzlösung in die Emboliezone eingespritzt, um damit den Embolus zu fragmentieren. Es folgt die sofortige Absaugung von Fragmenten, Gerinnseln und Restkochsalz mit dem Katheter. In einer kleinen Serie von 12 Patienten konnten 10 Patienten mit dieser Prozedur gerettet werden (Sterblichkeit 17\%) [15]. Über den Katheter kann dann direkt auch eine Thrombolyse appliziert werden [16]. Auch kleinlumige Katheter und seit kurzer Zeit verfügbare steuerbare Drähte mit einem Hohlraum eignen sich zur gezielten Applikation von Thrombolytika [17].

Rotationsembolektomie. Die Rotationskatheterfragmentierung führt zur Fragmentierung des Embolus mit oder ohne gleichzeitige Aspiration der Gerinnselfragmente $[18,19]$. In den meisten Fällen kann hiermit eine Rekanalisierung erzielt werden. In den vorliegenden Studien rangiert die Sterblichkeit bei dieser Therapieform zwischen 5 und 20\% [20].

Aspirationsembolektomie. Über einen großlumigen Zugang kann der Embolus mit einem Katheter mit Unterdruck abgesaugt werden. Einige Autoren kombinieren diese Art der Absaugung mit einer vorherigen Fragmentation mit Pigtailkatheter oder Ähnlichem [21,22].

Kathetergestützte Thrombolyse. Über in Pulmonalposition direkt proximal des Embolus platzierte Katheter kann lokal eine niedrig dosierte Thrombolyse appliziert werden (z. B. Actilyse). Die Effektivität der lokalen Lyse kann mechanisch oder durch Beschallung mit Ultraschall hoher Energie verstärkt werden. Diese Verfahren haben aber noch nicht Einzug in die klinische Routine gehalten.

\section{Antikoagulation}

Wichtigster Pfeiler der Therapie der Lungenembolie ist zweifelsfrei die plasmatische Antikoagulation in therapeutischer Dosierung. In der Akutphase sollte man unfraktioniertes Heparin nur dann verwenden, wenn in einer Hochrisikosituation oder bei Intermediärrisiko-Patienten eine Thrombolyse durchgeführt wird. Wegen besserer Evidenz sollte man in allen anderen Fällen entweder parenteral niedermolekulares Heparin oder Fondaparinux einsetzen (IA-Empfehlung) mit überlappender Einstellung auf einen Vitamin-K-Antagonisten (Ziel-INR: 2 - 3) oder ein direktes orales Antikoagulans (DOAK) wie Rivaroxaban, Dabigatran oder Apixaban (IB-Empfehlung) [1]. Im Vergleich zu Vitamin-K-Antagonisten sind DOAK bei der Verhinderung von Rezidiven einer venösen Thromboembolie nicht unterlegen, weisen aber ein deutlich reduziertes Blutungsrisiko auf. Für Patienten mit schwerer Niereninsuffizienz (GFR $<15 \mathrm{ml} / \mathrm{min}$ bei Rivaroxaban und Apixaban bzw. GFR $<30 \mathrm{ml} / \mathrm{min}$ bei Dabigatran) stehen DOAK jedoch nicht zur Verfügung.

Rivaroxaban. Der Faktor-Xa-Hemmer Rivaroxaban ist basierend auf dem EINSTEIN-Phase-3-Studienprogramm seit 2012 zugelassen und seither im klinischen Einsatz (Tab.4) [23]. Für die ersten 3 Wochen werden $2 \times 15 \mathrm{mg}$ verabreicht, gefolgt von der Erhaltungstherapie mit $1 \times 20 \mathrm{mg}$. Durch die Extensionsstudien (Abb.4) wurde in die Leitlinien bereits eine Therapieverlängerung mit den DOAK zur Sekundärprophylaxe der unprovozierten Lungenembolie um 6-12 Monate aufgenommen und für Patienten mit niedrigem Blutungsrisiko empfohlen (IIaB-Empfehlung) [1]. Rivaroxaban kann dann mit $1 \times 20 \mathrm{mg}$ für weitere 12 Monate gegeben werden [24].

\section{Tabelle 4}

Therapie der venösen Thromboembolie mit DOAK.

\begin{tabular}{|c|c|c|c|c|}
\hline & Europäische Zulassung & Initialdosis & Erhaltungsdosis & Sekundärprävention \\
\hline Rivaroxaban & 2012 & $\begin{array}{l}2 \times 15 \mathrm{mg} \\
\text { für } 21 \text { Tage }\end{array}$ & $1 \times 20 \mathrm{mg}$ & $\begin{array}{l}6-12 \text { Monate } \\
1 \times 20 \mathrm{mg}\end{array}$ \\
\hline Dabigatran & 2014 & niedermolekulares Heparin & $2 \times 150 \mathrm{mg}$ & $\begin{array}{l}\text { bis } 6 \text { Monate } \\
2 \times 150 \mathrm{mg}\end{array}$ \\
\hline Apixaban & 2014 & $\begin{array}{l}2 \times 10 \mathrm{mg} \\
\text { für } 7 \text { Tage }\end{array}$ & $2 \times 5 \mathrm{mg}$ & $\begin{array}{l}12 \text { Monate } \\
2 \times 2,5 \text { oder } 5 \mathrm{mg}\end{array}$ \\
\hline
\end{tabular}


Dabigatran. Seit dem Sommer 2014 ist auch der Thrombininhibitor Dabigatran zur Lungenembolietherapie zugelassen (Zulassungsstudie RE-COVER) [25]. Initiiert wird die Therapie mit Dabigatran $2 \times 150 \mathrm{mg}$, laut der Zulassung aber nicht direkt mit einer Monogabe des DOAK, sondern ähnlich wie bei Vitamin-KAntagonisten überlappend mit niedermolekularem Heparin. Nach Ablauf der Therapiedauer kann bei niedrigem Blutungsrisiko mit $2 \times 150 \mathrm{mg}$ um 6 Monate verlängert werden (Abb.4) [26].

Apixaban. Mit Apixaban ist seit Sommer 2014 ein weiterer Faktor-Xa-Hemmer zugelassen und kann ähnlich wie Rivaroxaban direkt (ohne Überlappung mit niedermolekularem Heparin) in der Dosierung von $2 \times 10 \mathrm{mg}$ über 7 Tage gegeben werden (Zulassungsstudie AMPLIFY) [27]. Es schließt sich die Erhaltungstherapie mit $2 \times 5 \mathrm{mg}$ an. Bei niedrigem Blutungsrisiko ist gemäß der AMPLIFY-EXT-Studie eine Sekundärprophylaxe für 12 Monate mit der Erhaltungsdosis $2 \times 5 \mathrm{mg}$ möglich (Abb.4) [28]. Mit Apixaban wurde neben der regulären Erhaltungsdosis auch die Therapieverlängerung mit halbierter Dosis untersucht $(2 \times 2,5 \mathrm{mg})$ und verhinderte Rezidive einer venösen Thromboembolie ähnlich effektiv, ohne das Blutungsrisiko zu erhöhen. Die Sekundärprävention mit $2 \times 2,5 \mathrm{mg}$ ist daher insbesondere für ältere Patienten oder solche mit reduzierter Nierenfunktion attraktiv. Sowohl Apixaban als auch Rivaroxaban können bei Niereninsuffizienz bis zu einer GFR von $30 \mathrm{ml} / \mathrm{min}$ gegeben werden (zulassungskonform sogar bis $15 \mathrm{ml} / \mathrm{min}$ möglich). Dabigatran wird zwar stärker renal metabolisiert, kann aber ebenfalls zulassungskonform bis zu einer GFR von $30 \mathrm{ml} / \mathrm{min}$ eingesetzt werden.

Therapiedauer. Zur regulären Therapiedauer geben die neuen Leitlinien etwas konkretere Hinweise als ältere Versionen, obwohl die Spannen immer noch recht groß sind und weiterhin ein wesentliches Gewicht auf individuellen Therapieentscheidungen liegt. Nach einer Lungenembolie durch eine tiefe Venenthrombose, die von einem transienten Faktor provoziert wurde (z.B. orthopädische Operation), sollte für 3 Monate behandelt werden (IB-Empfehlung) [1]. Nach unprovozierter Lungenembolie sollte man für mindestens 3 Monate eine Antikoagulation durchführen (IA-Empfehlung) und eine Verlängerung je nach Rezidiv- und Blutungsrisiko erwägen. Nach einem Rezidiv einer venösen Thromboembolie sollte dauerhaft antikoaguliert werden (IB-Empfehlung).

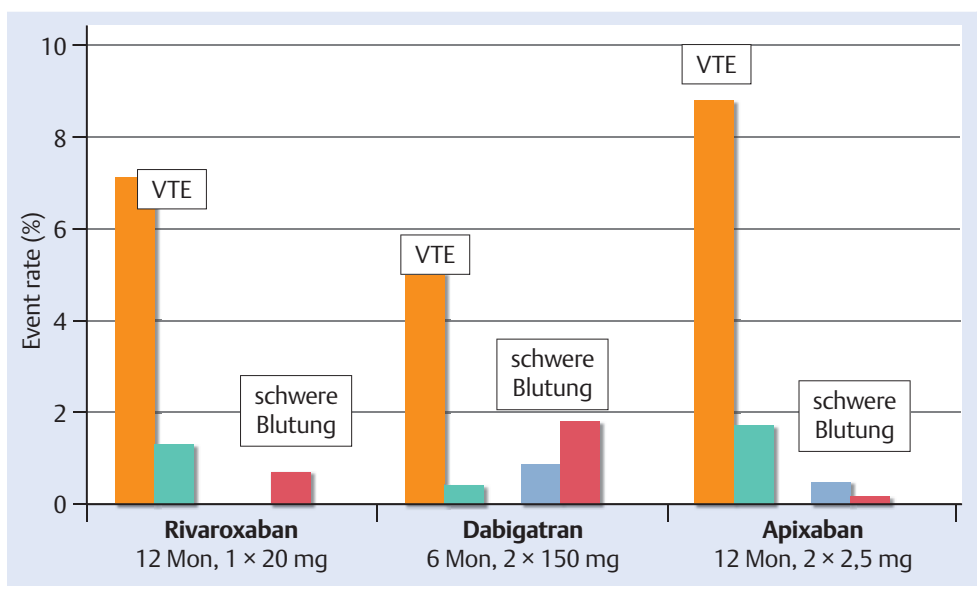

Abb.4 Raten der Rezidive einer venösen Thromboembolie und schwerer Blutungsereignisse in den Extensionsstudien der DOAK zur sekundärprophylaktischen Therapieverlängerung nach Ablauf der regulären Therapiedauer [24, 26, 28]. Orange und blau - Plazebo, grün und rot - DOAK.

Nach Beendigung der plasmatischen Antikoagulation kann, basierend auf den positiven Daten der WARFASAund ASPIRE-Studien, auch eine längerfristige Plättchenhemmung mit Azetylsalizylsäure (ASS) erwogen werden [29, 30]. Mit ASS lassen sich Rezidive einer venösen Thromboembolie dann effektiv verhindern, wenn eine therapeutische Antikoagulation wegen dem Überwiegen des Blutungsrisikos nicht mehr gerechtfertigt erscheint. Patienten mit einem Rezidiv einer venösen Thromboembolie sollten hingegen nicht mit ASS, sondern mit einer plasmatischen Dauerantikoagulation therapiert werden.

Für Patienten mit absoluter Kontraindikation für eine Antikoagulation sollte man beim Nachweis einer tiefen Venenthrombose in der unteren Extremität einen (passageren) V.-cava-Filter erwägen (IlaC-Empfehlung) [1]. Auch bei Rezidiven einer venösen Thromboembolie unter Antikoagulation mit therapeutischer INR oder plausibler täglicher DOAK-Einnahme sollte die Filterplatzierung erwogen werden.

DOAK sind ähnlich effektiv, aber deutlich sicherer als Vitamin-K-Antagonisten. Eine Therapieverlängerung sollte mit DOAK erwogen werden. Auch die Plättchenhemmung mit ASS verhindert Rezidive einer venöse Thromboembolie und kann für eine Dauertherapie in Betracht gezogen werden, wenn keine plasmatische Antikoagulation mehr erforderlich ist. 


\section{Kernaussagen}

- Hochrisikopatienten mit Lungenembolie sind hämodynamisch instabil und sollten umgehend einer pulmonalen Rekanalisierung zugeführt werden, um die hohe Letalität zu reduzieren.

- Therapie der Wahl ist in der Regel eine systemische Thrombolyse, alternativ sollte man eine chirurgische Embolektomie anstreben.

- Auch Intermediärrisiko-Patienten mit akuter Rechtsherzbelastung und einer Erhöhung der myokardialen Nekroseparameter profitieren von einer Lyse, wenn sie jünger als 75 Jahre sind, ein niedriges Blutungsrisiko haben und keine Kontraindikationen vorliegen.

- Zentraler Pfeiler der Lungenembolietherapie ist die sofortige plasmatische Antikoagulation in therapeutischer Dosierung.

- Neu zugelassen sind für Therapie und Sekundärprophylaxe die DOAK Rivaroxaban, Dabigatran und Apixaban, die ähnlich effektiv Rezidive einer venösen Thromboembolie verhindern, aber weniger Blutungskomplikationen verursachen als VitaminK-Antagonisten.

Interessenkonflikt: Daniel Dürschmied erhielt Sprecherhonorare von Lilly und Bayer.

Keine Interessenkonflikte bei den anderen Autoren.

\section{Über die Autoren}

\section{Daniel Dürschmied}

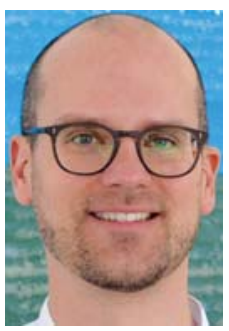

PD Dr. med. Jahrgang 1976. 1997 -

2004 Studium der Humanmedizin an der Universität Ulm. 2004-2012

Facharztausbildung als Internist und Kardiologe am Universitäts-Herzzentrum Freiburg - Bad Krozingen und Uniklinikum Freiburg. 2007-2009 wissenschaftliches Post-Doc an der Harvard Medical School in Boston, USA mit Schwerpunkt Thrombozytenfunktion. Seit 2012 Oberarzt der Kardiologie und Angiologie am UniversitätsHerzzentrum Freiburg - Bad Krozingen und der interdisziplinären internistischen Intensivmedizin am Uniklinikum Freiburg.

\section{Jürgen Heinz}

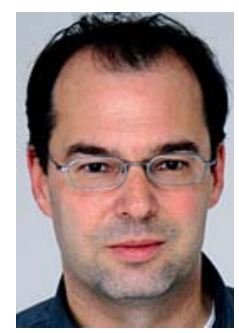

Dr. med. Jahrgang 1963. 1984 - 1991

Studium der Humanmedizin an der Albert-Ludwigs-Universität Freiburg. 1991 - 2003 Facharztausbildung zum Internisten und Facharzt für Hämatologie und Onkologie am Universitätsklinikum Freiburg. Seit 2004 Leiter der Gerinnungsambulanz der Klinik für Innere Medizin I (Hämatologie, Onkologie und Stammzelltransplantation) des Universitätsklinikums Freiburg.

\section{Matthias Siepe}

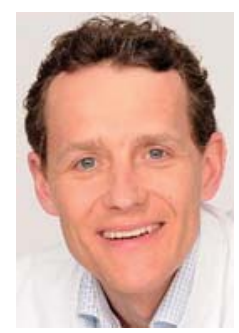

Prof. Dr. Jahrgang 1974. 1995-2002 Studium der Humanmedizin in Freiburg. 2003 und 2004 Forschungsaufenthalte in Paris und Bern. 2002 2010 Facharztausbildung zum Herzund Gefäßchirurgen in Freiburg. Dort auch Tätigkeit als Oberarzt (2011 - 2012) und leitender Oberarzt (seit 2012) mit den Spezialgebieten Kinderherzchirurgie, Aortenchirurgie und Herzunterstützungssysteme. Seit 2013 als Abteilungschefarzt der Herz- und Gefäßchirurgie am Standort Bad Krozingen des Universitäts-Herzzentrums tätig.

\section{Christoph Bode}

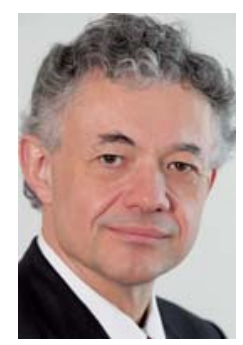

Prof. Dr. Er erwarb seine klinische und wissenschaftliche Reputation in Heidelberg und Harvard (Boston, USA). Er ist derzeitig Präsident der Gesellschaft für Thrombose und Hämostase, Chairman of the Assembly of International Governors (AIG) und Europäischer Herausgeber der Fachzeitschrift „Circulation“.

1999 übernahm er als ärztlicher Direktor die Abteilung Innere Medizin III der Universität Freiburg als Ordinarius.

\section{Korrespondenzadresse}

PD Dr. med. Daniel Dürschmied

Kardiologie und Angiologie I

Universitäts-Herzzentrum Freiburg - Bad Krozingen

Hugstetter Straße 55

79106 Freiburg

E-Mail: daniel.duerschmied@universitaets-

herzzentrum.de 


\section{Literatur}

1 Konstantinides SV, Torbicki A et al. 2014 ESC Guidelines on the diagnosis and management of acute pulmonary embolism. Eur Heart J 2014. Im Internet: http://eurheartj.oxfordjournals.org/content/35/43/3033

2 Kyrle PA, Minar E, Bialonczyk C et al. The risk of recurrent venous thromboembolism in men and women. $\mathrm{N}$ Engl J Med 2004; 350: $2558-2563$

3 Cook D, McMullin J, Hodder R et al. Prevention and diagnosis of venous thromboembolism in critically ill patients: a Canadian survey. Crit Care 2001; 5: 336-342

4 Bahloul M, Chaari A, Kallel H et al. Pulmonary embolism in intensive care unit: Predictive factors, clinical manifestations and outcome. Annals of thoracic medicine 2010; 5: 97-103

5 Davenport A. Heparin-induced thrombocytopenia during renal replacement therapy in the intensive care unit. Crit Care 2008; 12: 158

6 Seigerman M, Cavallaro P, Itagaki S et al. Incidence and outcomes of heparin-induced thrombocytopenia in patients undergoing cardiac surgery in North America: an analysis of the nationwide inpatient sample. Journal of cardiothoracic and vascular anesthesia 2014; 28: 98-102

7 Aujesky D, Obrosky DS, Stone RA et al. Derivation and validation of a prognostic model for pulmonary embolism. Am J Respir Crit Care Med 2005; 172: 1041 - 1046

8 Stein PD, Matta F. Pulmonary embolectomy in elderly patients. Am J Med 2014; 127: 348-350

9 Bloomfield P, Boon NA, de Bono DP. Indications for pulmonary embolectomy. Lancet 1988; 2: 329

10 Rosenberger P, Shernan SK, Mihaljevic T et al. Transesophageal echocardiography for detecting extrapulmonary thrombi during pulmonary embolectomy. The Annals of thoracic surgery 2004; 78: $862-866$; d6

11 Clarke DB, Abrams LD. Pulmonary embolectomy: a 25 year experience. Journal of thoracic and cardiovascular surgery 1986; 92: $442-445$

12 Yalamanchili K, Fleisher AG, Lehrman SG et al. Open pulmonary embolectomy for treatment of major pulmonary embolism. The Annals of thoracic surgery 2004; 77: 819-823; d23

13 Aklog L, Williams CS, Byrne JG et al. Acute pulmonary embolectomy: a contemporary approach. Circulation 2002; 105: $1416-1419$

14 Dauphine C, Omari B. Pulmonary embolectomy for acute massive pulmonary embolism. The Annals of thoracic surgery 2005; 79: $1240-1244$

15 Kuo WT, van den Bosch MA, Hofmann LV et al. Catheterdirected embolectomy, fragmentation, and thrombolysis for the treatment of massive pulmonary embolism after failure of systemic thrombolysis. Chest 2008; 134: 250-254
16 Koning R, Cribier A, Gerber L et al. A new treatment for severe pulmonary embolism: percutaneous rheolytic thrombectomy. Circulation 1997; 96: $2498-2500$

17 Witsch T, Mauler M, Herr $\mathrm{N}$ et al. A novel hollow and perforated flexible wire allows the safe and effective local application of thrombolytic therapy in a mouse model of deep vein thrombosis. J Thromb Thrombolysis 2014; 37: 450-454

18 Brady AJ, Crake T, Oakley CM. Percutaneous catheter fragmentation and distal dispersion of proximal pulmonary embolus. Lancet 1991; 338: 1186-1189

19 Schmitz-Rode T, Janssens U, Duda SH et al. Massive pulmonary embolism: percutaneous emergency treatment by pigtail rotation catheter. J Am Coll Cardiol 2000; 36: 375 - 380

20 Eid-Lidt G, Gaspar J, Sandoval J et al. Combined clot fragmentation and aspiration in patients with acute pulmonary embolism. Chest 2008; 134: 54-60

21 Engelberger RP, Kucher N. Catheter-based reperfusion treatment of pulmonary embolism. Circulation 2011; 124: 2139 2144

22 Cuculi F, Kobza R, Bergner M et al. Usefulness of aspiration of pulmonary emboli and prolonged local thrombolysis to treat pulmonary embolism. Am J Cardiol 2012; 110: 1841 - 1845

23 Buller HR, Prins MH, Lensin AW et al. Oral rivaroxaban for the treatment of symptomatic pulmonary embolism. N Engl J Med 2012; 366: $1287-1297$

24 Bauersachs R, Berkowitz SD, Brenner B et al. Oral rivaroxaban for symptomatic venous thromboembolism. N Engl J Med 2010; 363: 2499-2510

25 Schulman S, Kearon C, Kakkar AK et al. Dabigatran versus warfarin in the treatment of acute venous thromboembolism. N Engl J Med 2009; 361: 2342-2352

26 Schulman S, Kearon C, Kakkar AK et al. Extended use of dabigatran, warfarin, or placebo in venous thromboembolism. N Engl J Med 2013; 368: 709-718

27 Agnelli G, Buller HR, Cohen A et al. Oral apixaban for the treatment of acute venous thromboembolism. N Engl J Med 2013; 369: 799-808

28 Agnelli G, Buller HR, Cohen A et al. Apixaban for extended treatment of venous thromboembolism. N Engl J Med 2013; 368: 699- 708

29 Becattini C, Agnelli G, Schenone A et al. Aspirin for preventing the recurrence of venous thromboembolism. N Engl J Med 2012; 366: $1959-1967$

30 Brighton TA, Eikelboom JW, Mann K et al. Low-dose aspirin for preventing recurrent venous thromboembolism. N Engl J Med 2012; 367: 1979-1987

31 Meyer G, Vicaut E, Danays T et al. Fibrinolysis for patients with intermediate-risk pulmonary embolism. N Engl J Med 2014; 370: $1402-1411$ 


\section{CME-Fragen}

\section{CME.thieme.de}

\section{CME-Teilnahme}

- Viel Erfolg bei lhrer CME-Teilnahme unter http://cme.thieme.de

- Diese Fortbildungseinheit ist 12 Monate online für eine CME-Teilnahme verfügbar.

- Sollten Sie Fragen zur Online-Teilnahme haben, unter http://cme.thieme.de/hilfe finden Sie eine ausführliche Anleitung.

1

Häufig führt man bei Patienten mit akuter Lungenembolie noch während des initialen Aufenthalts auf der Intensivstation nach Therapieeinleitung ein Thrombophilie-Screening durch. Die Bestimmung welches der folgenden Parameter ist dabei sinnvoll?

\section{2}

Herr K. ist ein 56-jähriger Raucher mit akuter Dyspnoe. Sie stellen bei hämodynamischer Stabilität eine ausgedehnte Lungenembolie fest und registrieren echokardiografische Zeichen einer akuten Rechtsherzbelastung. Troponin ist erhöht. Wie schätzen Sie sein Risiko ein, in den nächsten Tagen bis Wochen an der Lungenembolie zu versterben?

\section{3}

Unter welchen Bedingungen wäre zur Therapie von Herrn K. eine systemische Thrombolyse sinnvoll?

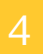

Welche Patienten sollten umgehend eine systemische Thrombolyse erhalten?
A Faktor-V-Mutation
B Faktor-II-Mutation
C Protein $\mathrm{C}$ und Protein $\mathrm{S}$
D Antithrombin
E keiner

5

In welchen Fällen sollte man die Platzierung eines Filters in der V. cava inferior erwägen?
A wenn eine Operation geplant wäre
B wenn eine Blutungsneigung bestünde
C wenn er über schwärzlichen Stuhl berichtete
D wenn keine Hinweise auf eine erhöhte Blutungsneigung vorlägen
E wenn Herr K. berichtete, dass vor Kurzem ein maligner Gehirntumor festgestellt wurde

A bei hämodynamischer Instabilität und nachgewiesener Lungenembolie ohne erkennbare Kontraindikationen

B bei Hypertonie, akuter Rechtsherzbelastung und vermuteter Lungenembolie

C unter Reanimation nach schwerem Autounfall mit Polytrauma

D bei hämodynamischer Stabilität und echokardiografischem Verdacht auf eine rechtskardiale Raumforderung ohne erkennbare Kontraindikationen

E bei Lungenembolie und Thrombophilie ohne Rechtsherzbelastung ohne erkennbare Kontraindikationen 


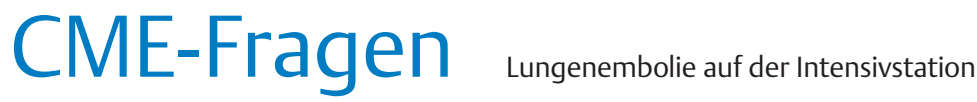

\section{6}

Welche Therapieoptionen sind bei Patienten mit Hochrisiko-Lungenembolie zur hämodynamischen Stabilisierung sinnvoll?

\section{7}

In welchen Fällen sollte chirurgisch embolektomiert werden?
A primär alleinige Dobutamingabe
B intravenöse Vasodilatatorengabe
C milde Volumengabe, Noradrenalin, ggf. Suprarenin
D aggressive Volumentherapie analog des Vorgehens bei hämorrhagischem Schock
E intravenöser Betablocker
A bei Hochrisiko-Lungenembolie und relativer Kontraindikation für eine Thrombolyse
B bei Niedrigrisiko-Lungenembolie und relativer Kontraindikation für eine Thrombolyse
C bei gutem Ansprechen auf eine initiale Lyse
D bei Niedrigrisiko-Lungenembolie und hohem Leidensdruck
E bei Hochrisiko-Lungenembolie und absoluter Kontraindikation für eine Thrombolyse

8

Welches Verfahren sollte generell während einer chirurgischen Embolektomie angewendet werden?

\section{9}

Welches Regime ist zur Lungenembolietherapie zugelassen?

\section{0}

Welcher Thrombozytenhemmer kann nach geplantem Absetzen einer plasmatischen Antikoagulation zur Therapie einer venösen Thromboembolie, basierend auf guten Studiendaten, eingesetzt werden?
A ECLS
B Kompressionssonografie der tiefen Venen
C begleitende intravenöse Thrombolyse
D transösophageale Echokardiografie
E Hirndrucksonde
A niedrig dosierte Tenecteplase
B Dabigatran initial $2 \times 110 \mathrm{mg}$, dann $2 \times 150 \mathrm{mg}$
C Rivaroxaban initial $2 \times 15 \mathrm{mg}$ für 21 Tage, dann $1 \times 20 \mathrm{mg}$
D initiale Gabe von Phenprocoumon als Monotherapie
E niedrig dosierte Alteplase bei Niedrigrisiko-Lungenembolie
A Ticagrelor
B ASS
C Cangrelor
D Prasugrel
E Eptifibatid 